\title{
Statistical approaches to uncertainty: $p$ values and confidence intervals unpacked
}

\author{
Introduction \\ What is statistical uncertainty? \\ Statistical uncertainty is the uncertainty (present even in a representative sample) associated with the use of sample data to make statements about the wider \\ population. \\ Why do we need measures of uncertainty? \\ It usually is not feasible to include all individuals from a target population in a single study. For example, in a randomised controlled trial (RCT) of a new treatment \\ for hypertension, it would not be possible to include all individuals with hypertension. Instead, a sample (a small subset of this population) is allocated to receive \\ either the new or the standard treatment

\section{What are the measures of uncertainty?} \\ Either hypothesis tests (with the calculation of p values) or confidence intervals ( $\mathrm{Cls}$ ) can quantify the amount of statistical uncertainty present in a study, though $\mathrm{Cls}$ \\ are usually preferred.
}

$\mathrm{n}$ a previous Statistics Note, we defined terms such as experimental event risk (EER), control event risk (CER), absolute risk reduction (ARR), and number needed to treat (NNT). ${ }^{1}$ Estimates such as ARR and NNT alone, however, do not describe the uncertainty around the results. P values and CIs provide additional information to help us determine whether the results are both clinically and statistically significant (table 1).

Table 1. Outcomes in the RCT comparing streptomycin with bed rest alone in the treatment of tuberculosis

\begin{tabular}{lllllll}
\hline Intervention & Survival & Death & Risk of death & ARR & P value & 95\% Cl \\
\hline Streptomycin $(n=55)$ & 51 & 4 & $4 / 55=7.3 \%$ (EER) & $25.9 \%-7.3 \%=19.6 \%$ & $5.7 \%$ to $33.6 \%$ & 0.006 \\
Bed rest $(n=52)$ & 38 & 14 & $14 / 52=25.9 \%$ (CER) & & \\
\hline
\end{tabular}

The ARR (the difference in risk) is estimated to be $19.6 \%$ with a $95 \%$ CI of $5.7 \%$ to $33.6 \%$. The p value of 0.006 means that an ARR of $19.6 \%$ or more would occur in only 6 in 1000 trials if streptomycin was equally as effective as bed rest. Since the $p$ value is less than 0.05 , the results are statistically significant (ie, it is unlikely that streptomycin is ineffective in preventing death). The $95 \%$ CI suggests that the likely true benefit of streptomycin could be as small as $5.7 \%$ or as large as $33.6 \%$, but is very unlikely to be $0 \%$ or less. Our best estimate for the ARR is $19.6 \%$ and hence the NNT is 6 (95\% CI 3 to 18 ). This means that we might have to treat as many as 18 people with

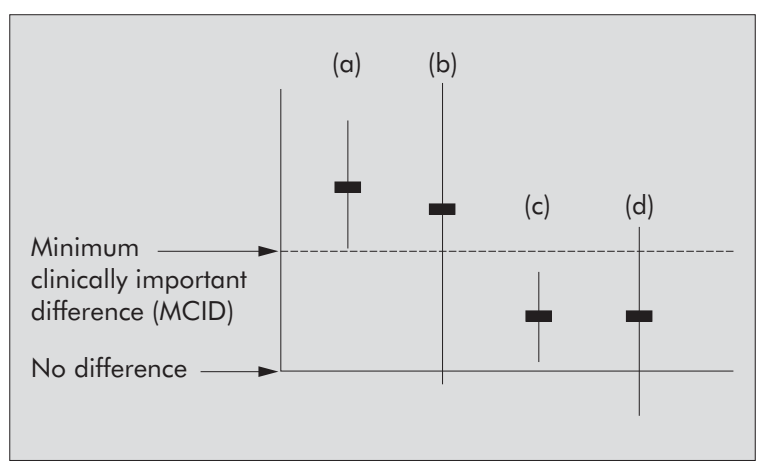

Figure 1 Clinical significance and statistical significance. streptomycin or as few as 3 to prevent 1 additional person dying of tuberculosis.

WHY IS THE $5 \%$ LEVEL ( $<<0.05)$ USED TO INDICATE STATISTICAL SIGNIFICANCE?

Conventionally, a p value of $<0.05$ is taken to indicate statistical significance. This $5 \%$ level is, however, an arbitrary minimum and $\mathrm{p}$ values should be much smaller, as in the above study $(\mathrm{p}=0.006)$, before they can be considered to provide strong evidence against the null hypothesis. Hence reporting the exact $\mathrm{p}$ value ( $\mathrm{eg}, \mathrm{p}=0.027$ ) is more helpful than simply stating that the result is significant at the 5\% level (or $1 \%$ level, as above).

\section{IF AN EFFECT IS STATISTICALLY SIGNIFICANT, DOES} THIS MEAN IT IS CLINICALLY SIGNIFICANT?

A statistically significant difference is not necessarily one that is of clinical significance. In the above example, the statistically significant effect $(p=0.006)$ is also clinically significant as even a modest improvement in survival is important. For many effects, however, the benefit needs to be somewhat greater than zero for it to be of clinical significance (ie, of sufficient benefit to be worth the effort of treatment). In figure 1, while both studies (a) and (c) show a statistically significant result, with the CIs not overlapping the "no difference" value, only (a) has a result that is consistent (in terms of the $\mathrm{CI}$ ) with at least a minimum clinically important difference (MCID). Studies (b) and (d) are not statistically significant, as their CIs overlap the values of no difference. 


\section{RELATED?}

While the 2 approaches to dealing with the problem of uncertainty are somewhat different, $\mathrm{p}$ values and CIs generally provide consistent results. If the effect is statisti- cally significant (at the 5\% level), then the 95\% CI will not include the value of "no difference", and vice versa. While CIs are preferable to $\mathrm{p}$ values in summarising study results, both approaches are commonly used.

Comparison of the use of $p$ values and confidence intervals in statistical inference

\begin{tabular}{ll}
\hline$P$ values and hypothesis tests & Confidence intervals \\
\hline What are they used for? &
\end{tabular}

$p$ values are used to assess whether a sample estimate is significantly different from a hypothesised value (such as zero-ie, no treatment effect). Hypothesis tests assess the likelihood of the estimate under the null hypothesis of no difference between 2 population values (or no treatment effect). Conventionally, if $p<0.05$, the null hypothesis is rejected.

What do they tell us?

The $p$ value is the probability that the observed effect (or more extreme ones) would have occurred by chance if in truth there is no effect.

However, it doesn't tell us anything about the size of the true effect and, moreover, since hypothesis tests are 2 tailed (we are interested in differences in either direction) it doesn't even tell us the direction of this effect. Thus, in the above example, the $p$ value of 0.006 indicates that an effect of $19.6 \%$ or more, in favour of either streptomycin or bed rest, would occur in only 6 in 1000 trials if in truth there is no effect.

\section{When can they be used?}

There are many different types of hypothesis test, each suitable for a particular type of data. For example, parametric tests (such as t-tests) are only suitable for large samples and for data that are drawn from a population which is approximately normally distributed.

\section{How are they calculated?}

The observed effect together with a measure of its variability (such as the standard error, SE) is used to calculate a "test statistic" (eg, t, $z, \chi^{2}$ ).

For example, a t statistic is calculated by dividing the observed effect by its SE. The value of the test statistic is used (from statistical tables) to determine the $p$ value.

Thus, in the example above (where $\operatorname{SE}(A R R)=7.1 \%$ ), the $z$ statistic (assuming that the ARR is approximately normally distributed) for the test of whether the risk of death differs between those allocated to streptomycin and those allocated to bed rest is calculated as $z=19.6 / 7.1=2.76$. This has an associated $p$ value of 0.006 .
Confidence intervals $(\mathrm{Cls})$ present a range of values around the sample estimate within which there is reasonable confidence that the true, but unknown, population value lies. The $95 \% \mathrm{Cl}$ (the range of values within which there is $95 \%$ probability that the true value lies) is most commonly used. It corresponds with the typical $5 \%$ significance level used in hypothesis tests.

The $\mathrm{Cl}$ provides a range of values whose limits are, with specified probability (typically 95\%), the smallest and the largest true population values consistent with the sample data. $\mathrm{A} \mathrm{Cl}$ can thus effectively function as a hypothesis test for an infinite number of values: if the $\mathrm{Cl}$ includes any 1 of these values then the sample estimate is not statistically significantly different from it. The $95 \% \mathrm{Cl}$ is of particular relevance to evidence-based practice (EBP), providing valuable information such as whether the interval includes or excludes clinically significant values.

$\mathrm{A} \mathrm{Cl}$ can, and should, be calculated for most measures of effect, such as differences between means (such as scores or weights), and differences in proportions, EER and CER, ARR, NNT, risk ratios (RR), and odds ratios (OR).

To calculate a $\mathrm{Cl}$ around a sample estimate, only 3 pieces of information are needed: the sample size ( $n$ ), the sample standard deviation (SD), and the " $z$ score," which varies depending on the degree of confidence wanted $195 \%, 99 \%$ etc). For a $95 \% \mathrm{Cl}, \mathrm{z}=1.96$, and for a $99 \% \mathrm{Cl}, \mathrm{z}=2.58 . \mathrm{A} 95 \% \mathrm{Cl}$ is calculated as:

Sample estimate \pm 1.96 standard errors (SE) of the measure (note: $\mathrm{SE}=\mathrm{SD} /{ }_{\sqrt{ }} \mathrm{n}$ ) Thus, in the above example (where SE(ARR) $=7.1 \%$ ), the $95 \% \mathrm{CI}$ for the true ARR is calculated as:

$19.6 \% \pm 1.96(7.1 \%)=5.7 \%$ to $33.6 \%$.

\section{WHY DOES SAMPLE SIZE HAVE TO BE CONSIDERED WHEN INTERPRETING THE SIZE OF THE P VALUE \\ AND THE WIDTH OF THE CI?}

The larger the sample, the less the uncertainty, the narrower the CI, and hence the smaller the observed effect that can be declared statistically significant $(\mathrm{p}<0.05)$. Thus, if a sample is very large, even a very small difference (which may be of no clinical relevance) may be statistically significant (see (c) in figure 1). The width of a CI is affected by both the sample size (n) and the sample SD. The larger the sample (and the smaller its variability), the greater the accuracy of the sample estimate and thus the narrower the CI. A wide CI can thus reflect either a small sample or one with large variability (see (b) in figure 1 ).

\section{CAN THE CONCLUSIONS FROM A HYPOTHESIS TEST BE IN ERROR?}

Since hypothesis tests are based on estimates of probability, their conclusions can be in error. There are 2 types of error: rejecting the null hypothesis when it is true (type I error; the probability of this error is 5\% if the 5\% significance level is used) and failing to reject the null hypothesis when it is false (type II error; the probability of this error is 1 - Power) (figure 2). Power and sample size will be discussed in more detail in a future Statistics Note.

\begin{tabular}{l|cc}
\hline \multirow{2}{*}{$\begin{array}{l}\text { Statistical } \\
\text { decision }\end{array}$} & $\begin{array}{c}\mid c \\
\text { Null hypothesis } \\
\text { true }\end{array}$ & $\begin{array}{c}\text { Null hypothesis } \\
\text { false }\end{array}$ \\
\cline { 2 - 3 } $\begin{array}{l}\text { Reject null } \\
\text { hypothesis }\end{array}$ & Type I error & Correct (power) \\
$\begin{array}{l}\text { Do not reject } \\
\text { null hypothesis }\end{array}$ & Correct & Type II error \\
\hline
\end{tabular}

Figure 2 Type I and II errors.

HELEN DOLL, BSc, DIP APP STATS, MSC Department of Public Health, University of Oxford, Oxford, UK

STUART CARNEY, MB, ChB, MPH, MRCPsych

Department of Psychiatry, University of Oxford, Oxford, UK 1 Carney S, Doll H. Evidence-Based Medicine 2005;10:102-3. 